Vol. 5, No. 1, 2020

\title{
STUDY OF THE PROPERTIES OF ANP FERTILIZER ENCAPSULATED WITH THE USE OF MODIFIED WASTE OF PET
}

\author{
Oleh Nahurskyy, Myroslav Malovanyy, Serhiy Synelnikov, Ivan Tymchuk, Galyna Krylova \\ Lviv Polytechnic National University, \\ 12, S. Bandery Str., Lviv, 79013, Ukraine \\ o_nagurskyy@ukr.net
}

https://doi.org/10.23939/ep2020.01.035

Received: 31.01 .2020

(C) Nahurskyy O., Malovanyy M., Synelnikov S., Tymchuk I., Krylova G., 2020

\begin{abstract}
The experimental investigation of the solubility of the prolonged-action nitroamophos obtained with the use of modified polyethylene terephthalate (PET) waste is presented. The kinetic coefficients of the diffusion process of the substances inside the shell are determined. The possibility of applying a theoretical model to predict the agro-ecological properties of encapsulated ammonium nitrate phosphate is demonstrated.
\end{abstract}

Key words: encapsulated ANP fertilizer, dissolution, kinetics, PET waste.

\section{Introduction}

Synthetic granular mineral fertilizers are used extensively in agriculture to compensate for the loss of plant nutrients in the soil. Due to soil fertilization, agricultural production increases by 30-40 \%. A longterm analysis, according to FAO, shows a clear upward trend in fertilizer use in the world. Thus, for the period from 2002 to 2012 , this increase was $3.38 \mathrm{~kg} / \mathrm{ha}$ of arable land; and in 2020 it is predicted to be $165 \mathrm{~kg} / \mathrm{ha}$ of arable land $[1,2]$.

Taking into account that the efficiency of fertilizer assimilation by plants varies between 40-60\%, the considerable amount of their loss is evident, which leads to environmental pollution. Many scientific works are devoted to reducing unproductive losses of mineral fertilizers. One of the effective ways to increase the amount of nutrients assimilated by plants and to prevent contamination of the environment with mineral fertilizers, is the application of fertilizers with prolonged action, in particular encapsulated ones [3-6]. However, the cost of long-action mineral fertilizers is considerably higher(by $500 \%$ or more) if compared to traditional ones. It is the main reason they are not widely used in agricultural production. To create encapsulated fertilizer available for mass consumption, the research regarding the use of plastic waste as the basis of a film-forming composition is of current interest.

The purpose of the research was to establish the regularities of nutrients release from granular synthetic fertilizers, encapsulated with a shell based on polyethylene terephthalate(PET) wastes.

\section{Materials and methods}

A granular complex ammonium nitrate phosphate (ANP) fertilizer encapsulated with a film-forming composition based on modified polyethylene terephthalate was used in the research. The modification of the primary processing wastes was carried out according to the method described in [7]. As a result, a substance was obtained, which was characterized by the ability to adhere to solid surfaces. For encapsulation by the method of shell applying on the layer of solid particles in the fluidized state, an ethyl acetate-based solution was prepared with a ratio of 5:1. The weight of the shell was $10 \%$ of the fertilizer weight. During encapsulation, the natural sorbent zeolite (in the form of a fine solid phase) was periodically fed to the layer to avoid sticking of the fertilizer granules. As a result, a layer of the shell that contained solid sorbent particles was formed on the surface of the fertilizer granules. Natural sorbents are widely used in agricultural production to improve soil structure, absorb harmful substances [8, 9], etc.

The study of the properties of the slow-release synthetic mineral fertilizer is carried out by different 
methods. The main difference between the methods is the environment in which the fertilizers under study are placed. Thus, according to the requirements of European standard EN 13266:2001, the study must be carried out in distilled water under constant stirring. The time of the complete release of the nutrients from the fertilizer granule is determined as the duration of this fertilizer action. However, the conditions of fertilizer dissolution in the soil medium differ from the pure water environment, which significantly affects the time of the complete release of the nutrients. Therefore, to characterize fertilizers of prolonged action, the investigations are carried out in the soil medium [10]. This is particularly true of the fertilizers, whose mechanism of release depends on chemical and biological processes [11]. In the case of the polymer coating, the temperature is a single external determinant of the nutrient release. The studies of the kinetics of the nutrient release were performed for a single particle in an aqueous medium under continuous stirring at the constant temperature by a conductometric method based on the measurement of the electrical conductivity of solutions.

\section{Results and discussions}

The studies were carried out with single particles of encapsulated ANP fertilizer. The volume of the solvent (distilled water) was $200 \mathrm{ml}$. To obtain the most accurate results, we selected particles of the correct geometric shape with the same weight. The averaged values of 5 parallel experiments are shown graphically in Fig. 1.

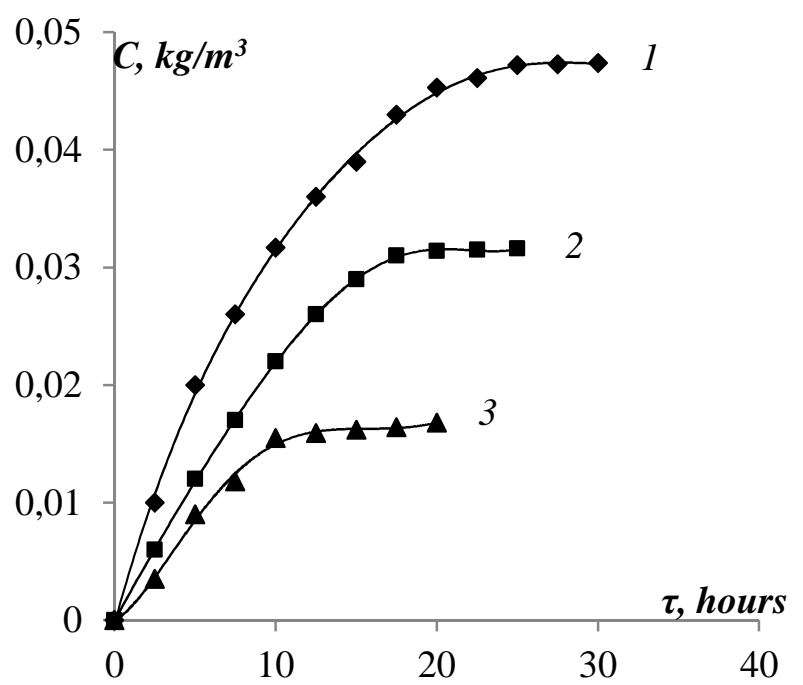

Fig. 1. . Kinetics of the nutrient release from the particles of encapsulated ANP fertilizer of different weight, mg: $1-29,2-20,3-11$

The experimental curves are correlated with the data obtained for other types of encapsulated fertilizers [12]. This allows concluding that it is possible to create mineral fertilizers encapsulated with modified PET and addition of a fine sorbent with the predicted duration of action. The experimental confirmation of such characteristics needs a long time. Taking into account the fertilizer characteristics declared by the manufacturer, the experimental studies can take several months. To significantly reduce the experimental time, the theoretical calculations of the process, using mathematical dependencies, may be used. The model of the release of the components from a single encapsulated particle of spherical shape is presented in [13]:

$$
\begin{gathered}
{\left[\left(1+2 \frac{\mathrm{Bi}+1}{\mathrm{Sh}}\right) \frac{\mathrm{k}}{3 \mathrm{a}}-\frac{1}{3 \mathrm{bk}}\right] \times} \\
\times\left[\frac{1}{2} \ln \frac{(\mathrm{k}+\varphi)^{2}\left(\mathrm{k}^{2}-\mathrm{k}+1\right)}{\left(\mathrm{k}^{2}-\mathrm{k} \varphi+\varphi^{2}\right)(\mathrm{k}+1)^{2}}+\sqrt{3} \operatorname{arctg} \frac{\sqrt{3} \mathrm{k}(\varphi-1)}{2\left(\mathrm{k}^{2}+\varphi\right)-\mathrm{k}(\varphi+1)}\right]=3 \mathrm{Fo}^{2}
\end{gathered}
$$

where

$$
\frac{\mathrm{r}}{\mathrm{R}}=\varphi ; \mathrm{m}=\frac{3 \mathrm{~W}}{4 \pi \mathrm{R}^{3}} ; \quad \alpha=\frac{\mathrm{C}_{\mathrm{s}}}{\rho_{\mathrm{s}}} ; \mathrm{a}=\alpha-\frac{1}{\mathrm{~m}}(1-\alpha) ; \mathrm{b}=\frac{1}{\mathrm{~m}}(1-\alpha) ; \mathrm{k}=\sqrt[3]{\frac{\mathrm{a}}{\mathrm{b}}} ;
$$

Fo, Sh, Bi are Fourier, Sherwood, Bio criterion, respectively.

$$
F o=\frac{\tau D_{1}}{R^{2}} ; \quad S h=\frac{\beta R}{D_{1}} ; B i=\frac{\beta \delta}{D_{2}} ;
$$

$\rho_{\mathrm{s}}$ is the density of the solid phase, $\mathrm{kg} / \mathrm{m}^{3}$; W is the solvent volume, $\mathrm{m}^{3} ; \mathrm{R}$ is the radius of the solid phase at the initial moment of the process, $\mathrm{m} ; \mathrm{r}$ is the radius of the solid phase that dissolves at any moment of the process, $\mathrm{m} ; \mathrm{C}_{\mathrm{s}}$ is the saturation concentration of the substance at the boundary of dissolution, $\mathrm{kg} / \mathrm{m}^{3} ; \delta$ is the thickness of the shell, $\mathrm{m} ; \mathrm{D}_{1}$ is the diffusion coefficient of the componentin the capsule volume, $\mathrm{m}^{2} / \mathrm{s} ; \mathrm{D}_{2}$ is the diffusion coefficient of the compound in the middle of the shell, $\mathrm{m}^{2} / \mathrm{s} ; \beta$ is the mass transfer of the component from the outer surface to the solvent medium, $\mathrm{m} / \mathrm{s} ; \tau$ is the process time, s.

Using this model and the above-mentioned kinetics of the release of the compounds (Fig. 1), the coefficient of internal diffusion in the polymer shell D2 was determined. This coefficient characterizes the permeability of the shell and is most accurately determined by the experimental results. For this purpose, the main mass transfer equation was used [12]:

$$
\mathrm{M}=\mathrm{kF}_{\mathrm{n}}\left(\mathrm{C}_{\mathrm{s}}-\mathrm{C}_{1}\right) \tau,
$$

where $\mathrm{k}$ is the mass transfer coefficient, $\mathrm{m} / \mathrm{s}$. 


$$
\mathrm{k}=\frac{1}{\delta / \mathrm{D}_{2}+1 / \beta} .
$$

To determine the diffusion coefficient inside the shell the Eq. $(3,4)$ are used:

$$
\mathrm{D}_{2}=\frac{\delta}{1 / \mathrm{k}-1 / \beta} .
$$

The mass transfer coefficient $\mathrm{k}$ is determined from Eq. (1) based on the experimental data. The radius of solid particle $\mathrm{R}$ is expressed through its weight.

$$
\mathrm{R}=\sqrt[3]{\frac{3}{4} \cdot \frac{\mathrm{M}_{\mathrm{u}}}{\pi \rho_{\mathrm{s}}}} .
$$

The shell thickness $\delta$ is expressed through the weight of shell $\mathrm{M}_{\mathrm{n}}$ :

$$
\delta=\frac{\mathrm{M}_{\mathrm{n}}}{4 \pi \mathrm{R}^{2} \rho_{\mathrm{n}}},
$$

where $\rho$ is the density of the shell material, $\mathrm{kg} / \mathrm{m}^{3}$.
Other necessary values are reference data or are determined by direct measurements. For the calculations, we chose experimental values on the straight-line plot of the kinetic curve shown in Fig. 2. Such an approach allows avoiding the effect of the component diffusion from the dissolution surface to the shell, which increases with the increase in the distance from the solid phase surface to the inner boundary of the capsule. In Fig. 2, this effect is indicated by the logarithmic nature of the kinetic curve. The initial data and the results of the calculations are shown in Table 1.

Using the above kinetic coefficients, we carried out the theoretical calculation according to Eq. (1) for experimental conditions. Comparison of the theoretical and experimental results for ANP fertilizer particles of different weight encapsulated with PET-based shell are shown in Fig. 3.

Table 1

Kinetic diffusion coefficients of ANP fertilizer components released from a polymeric capsule

\begin{tabular}{|c|c|c|c|c|c|c|}
\hline № & $\mathrm{M}_{\mathrm{Y}} \times 10^{6}, \mathrm{~kg}$ & $\mathrm{Mn} \times 10^{6}, \mathrm{~kg}$ & $\delta \cdot 10^{6}, \mathrm{~m}$ & $\mathrm{k} \times 10^{8}, \mathrm{~m} / \mathrm{s}$ & $\mathrm{D}_{2} \times 10^{12}, \mathrm{~m}^{2} / \mathrm{s}$ & $\bar{D}_{2} \times 10^{12}, \mathrm{~m}^{2} / \mathrm{s}$ \\
\hline 1. & 29 & 2.9 & 0.00021 & 1.52311 & 3.16 & \\
\cline { 1 - 5 } 2. & 20 & 2.0 & 0.00016 & 1.65161 & 2.65 & 2,80708 \\
\cline { 1 - 5 } 3. & 11 & 1.1 & 0.00014 & 1.8333 & 2.61 & \\
\hline
\end{tabular}

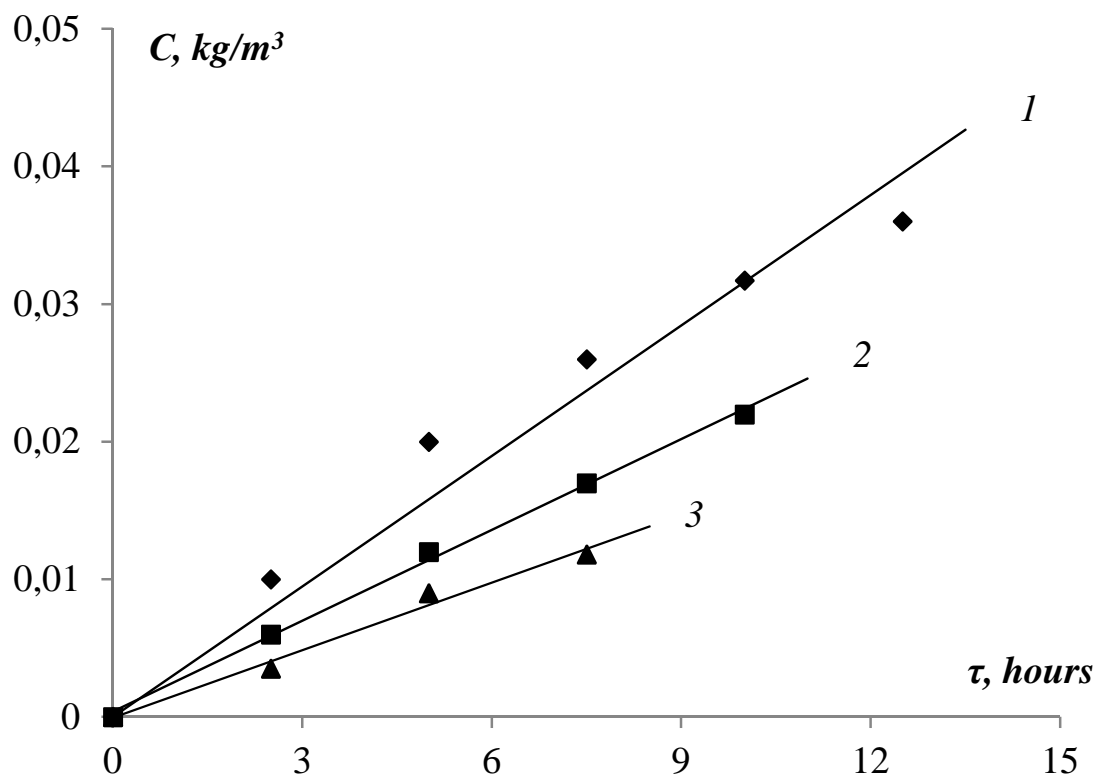

Fig. 2. Graphic dependences of the kinetics of the release of the components from the particles of encapsulated ANP fertilizer of different weight (mg) used to determine the diffusion coefficient $\mathrm{D}_{2}$ : 1-29, 2-20, 3-11 


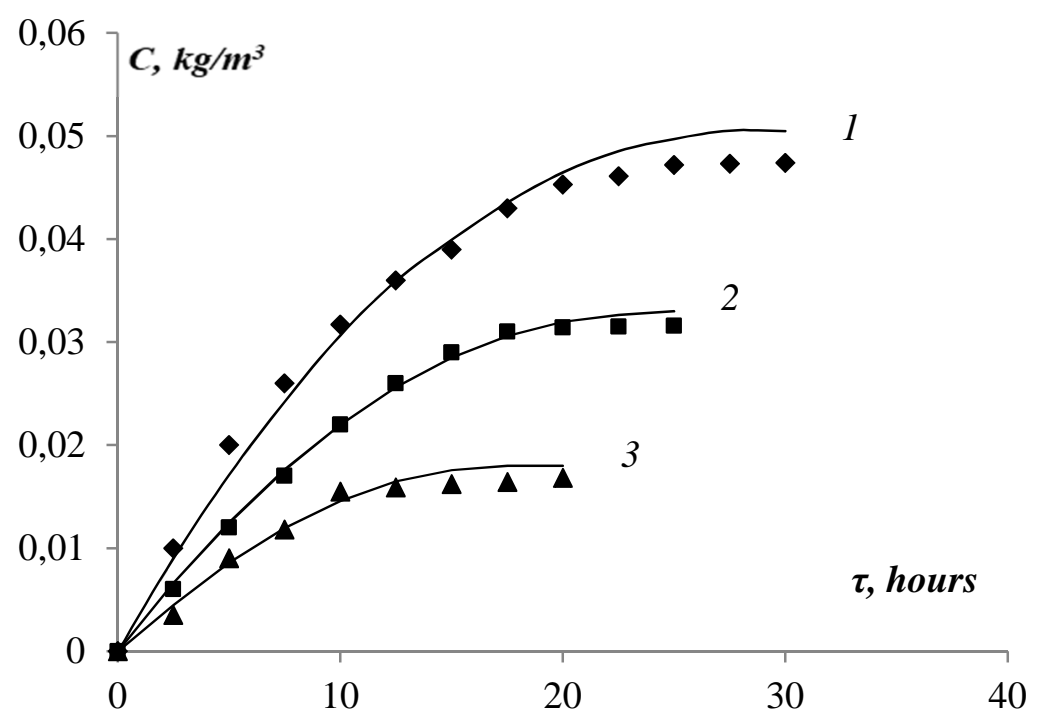

Fig. 3. Comparison of the theoretical (line) and experimental (point) kinetics of the release of mineral nutrients from the encapsulated ANP fertilizer particles of different weight, mg: 1-29, 2-20, 3-11

The relative error of the experimental and theoretical values is in the range of $3.0 \div 15.1 \%$. This confirms the adequacy of the applied mathematical model of the release of mineral nutrients from the ANP fertilizer particle encapsulated with PET-based film and the determination of the diffusion coefficient inside the shell using Eq. (2-6).

\section{Conclusions}

The experimental and theoretical studies of the properties of the granulated ANP fertilizer encapsulated with the PET-based film have made it possible to determine the kinetic parameters of the nutrients release. The adequacy of the used mathematical model was confirmed. The model allows predicting with sufficient accuracy the properties of encapsulated ANP fertilizer, depending on the shell weight without long-term experimental studies.

\section{References}

[1] Kucher A., Kucher L.: Golov. zhur. z pytan' agrobiz., 2017.

[2] Kernasjuk Ju.: Agrobiz. s'ogodni, 2017, 22 (365).

[3] Loginova I. V.: Zbir. nauk. prac' VNAU. Zemlerobstvo, 2011, 9(49),25.

[4] Bykin A. V., Bordjuzha N. P., Tarasenko O. V., Bordjuzha I. P.: Visn. HNAU Agrohimija 2013, 1, 100.

[5] Furdychko O. I.: Agroekologija. Agrarna nauka, Kyiv 2014.

[6] Pryjmak V. V.: Sel's'koe hazjajstvo, 2018, 3, 10, 70.

[7] Hak V. .. PhDthesis, Lviv Polytechnic Nats. Univ., Lviv 2011.

[8] Kuz'menko Je. I.: Agrohim. ta zemlerob., 2012, 15, 3, 12.

[9] Gurec' L. L., Capko Ju. L., Mal'ovanyj M. S., Vakal V. S.: Naukov. visn. NLTU Ukrajiny, 2019, 29, 2, 94.

[10] https://www.haifa-group.com/articles/slow-releasefertilizer-vs-controlled-release-fertilizer

[11] Nagursky O. A., Gumnyc'kyj Ja. M.: Voprosy hymyy y hymych. tehnol., 2012, 2, 202.

[12] Nagursky O.: Chem.\&Chem. Technol. 2012, 6, 1, 101. 